\title{
HDR Brachitherapy for Basal Cell Carcinoma
}

\author{
Lancellotta $\mathbf{V}^{1^{*}}$, Lupattelli $\mathbf{M}^{2}$, Zucchetti $\mathrm{C}^{3}$, Saccia $\mathrm{S}^{3}$, Cavalli $\mathrm{A}^{3}$ and Aristei $\mathrm{C}^{3}$ \\ ${ }^{1}$ Radiation Oncology Section, University of Perugia, Italy \\ ${ }^{2}$ Radiation Oncology Division, Santa Maria della Misericordia Hospital Perugia, Italy \\ ${ }^{3}$ Radiation Oncology Section, University of Perugia and Santa Maria della Misericordia Hospital, Italy
}

*Corresponding author: Valentina Lancellotta, Radiation Oncology Section, University of Perugia, Italy, Tel: +39 0755783614; E-mail: valentinalancellotta@libero.it Received date: Sep 30, 2015, Accepted date: Oct 29, 2015, Publication date: Nov 04, 2015

Copyright: (c) 2015 Lancellotta V, et al. This is an open-access article distributed under the terms of the Creative Commons Attribution License; which permits unrestricted use; distribution; and reproduction in any medium; provided the original author and source are credited.

\section{Abstract}

Aim: to evaluate tumor control, toxicity and cosmesis in patients with relapse of basal cell carcinoma (BSC) who underwent high-dose-rate brachytherapy (HDR-BT)using a regimen of hypofractionation.

Case presentation: A 93-year-old male, Karnosky Performace Status 90\%, was diagnosed to have a relapse of BSC. A biopsy confirmed a relapse of BSC. The patient underwent HDR-BT with 192 Ir. The prescription dose of 50 Gy in 20 fractions of 2.5 Gy was delivered daily. The Common Terminology Criteria for Adverse Events (CTCA v4.0) toxicity scale was used to asses acute toxicity and the Radiation Therapy Oncology Group (RTOG) and the European Organization for Research and Treatment of Cancer (EORTC) scale was used to asses late toxicity related to cosmesis.

The treatment was well tolerated. Clinically, the lesion completely regressed.

The skin acute toxicity was grade 2 CTCA v4.0 and late toxicity grade 1EORTC. When the patient was consulted about his cosmetic result, he was satisfied.

At the median follow-up of 7 months, the patient was alive and disease-free.

Conclusion: the HDR-BT can be an effective and safe treatment option for selected elderly patients with relapse skin cancer.

Keywords: Skin cancer; HDR-BT; Skin brachytherapy; Thermoplastic mask

\section{Introduction}

BSC is the most common skin cancer, representing $80-90 \%$ of all non melanoma skin cancer $[1,2]$. Surgery and radiotherapy are the first treatment for BSC, while topical therapy should be reserved for those patients where surgery and radiotherapy is contraindicated or impractical. The choice of appropriate therapy mainly depends on the age, performance status, lesion size and location, hystologic subtype and patient comorbidities [3].

Surgery, generally, is the first choice because it is a simple and effective procedure [4]. Radiotherapy (RT) is a viable alternative to surgery in selected cases, particularly in elderly patients [1].

Very few randomized studies have been undertaken to compare treatment modalities, although a recent meta-analysis concluded that surgery and RT are both effective, safe and have the lowest rates of recurrence [4].

HDR-BT is an alternative option to external beam RT and surgery in selected patients. It uses a192 Ir HDR radioactive source placed near the lesion, which emits radiation. It can be delivered with dedicated skin applicators [5]. The purpose of this case report is to asses toxicity, cosmesis and tumor control in a patient with BSC treated with HDRBT using a hypofractionational schedule.

\section{Case Presentation}

A 93-year-old male, Karnosky Performace Status 90\%, was diagnosed to have relapse of the BSC. The patient has already undergone a local treatment in 2008 (two operations for tumor resection and adjuvant external beam RT total dose 54 Gy in 27 fractions).

The examination showed an ulcerated lesion at the level of the eyebrow region of approximately $4 \mathrm{~cm}$ and a biopsy confirmed a relapse of BSC. The radiation oncologist contoured the Gross Tumor Volume (GTV) lesion on the patient's skin.

A moldable applicator, composed of a thermoplastic mask and hollow HDR-BT treatment catheters spaced at $1 \mathrm{~cm}$ intervals, were applied to the skin surface. The GTV lesion was contoured also on the thermoplastic mask and radiopaque landmarks were placed on the counturing (Figure 1).

Computed tomography $(\mathrm{CT})$ data were acquired with $0.3 \mathrm{~cm}$ slice thickness. All CT scans were transmitted to the Oncentra (Brachy Treatment Planning-Elekta) and one radiation oncologist contoured the GTV with the help of landmarks. A radial margin of $2 \mathrm{~cm}$ was added to create the clinical target volume (CTV). 


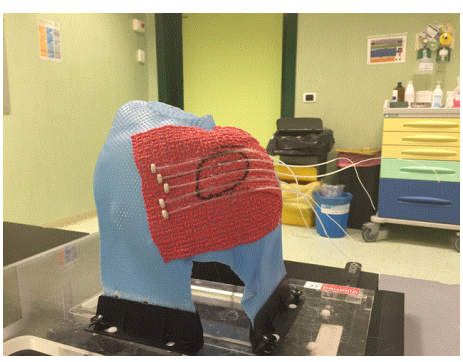

Figure 1: Termoplastic mask with catheters.

A single radioactive seed of the Iridium 192 was then directed to dwell positions within the hollow catheters. The location of the dwell positions and the amount of time the radioactive source stayed at each position were computer optimized to provide the most uniform radiation dose to the CTV. The CTV coverage and Dose Volume Histogram were reported in Figure 2.

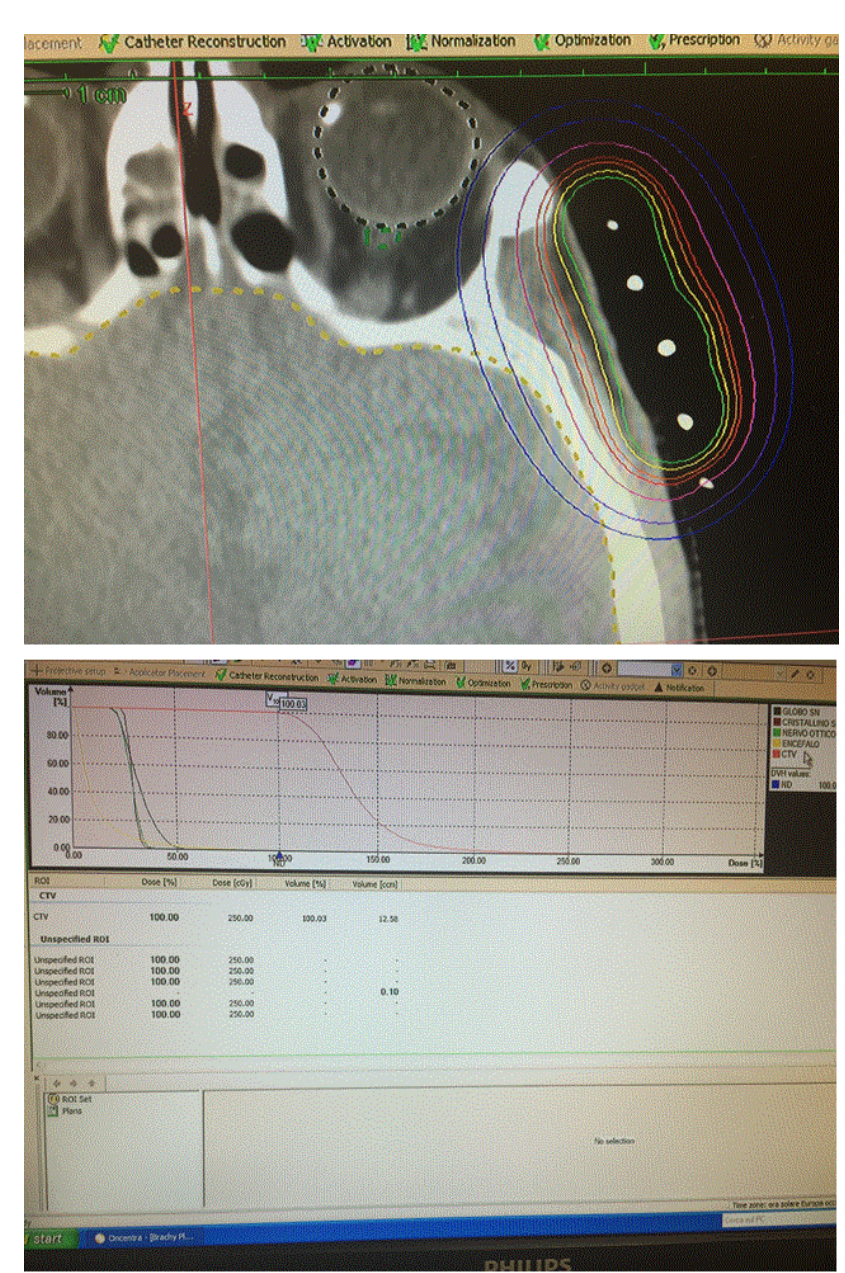

Figure 2: Coverage PTV and DVH.

The prescription dose of 50 Gy in 20 fractions of 2.5 Gy was delivered daily. Treatment duration was less than 5 minutes for all fractions. After treatment the patient was instructed to avoid sun exposure and to apply sunscreen. Patient was seen for follow-up at 2 and 4 weeks after brachytherapy to asses skin toxicity and at 3 and 6 months to evaluate efficacy and cosmesis. Common Terminology Criteria for Adverse Events (CTCA v4.0) toxicity scale [6] was used to asses acute toxicity and the Radiation Therapy Oncology Group (RTOG) and the European Organization for Research and Treatment of Cancer (EORTC) scale [7] was used to asses late toxicity related to cosmesis.

The treatment was well tolerated. Toxicity observed during treatment started after the $8^{\text {th }}$ fraction and was most severe after the $11^{\text {th }}$ fraction. The skin acute toxicity was grade 2 CTCA $v 4.0$, and was resolved with a topical treatment at the last 4 weeks of HDR-BT. We observed, for late toxicity, skin atrophy with hypopigmentation grade 1 EORTC. When patient was consulted about his cosmetic result, he was satisfied (Figure 3). At the last follow-up, the patient was alive and clinically disease-free.

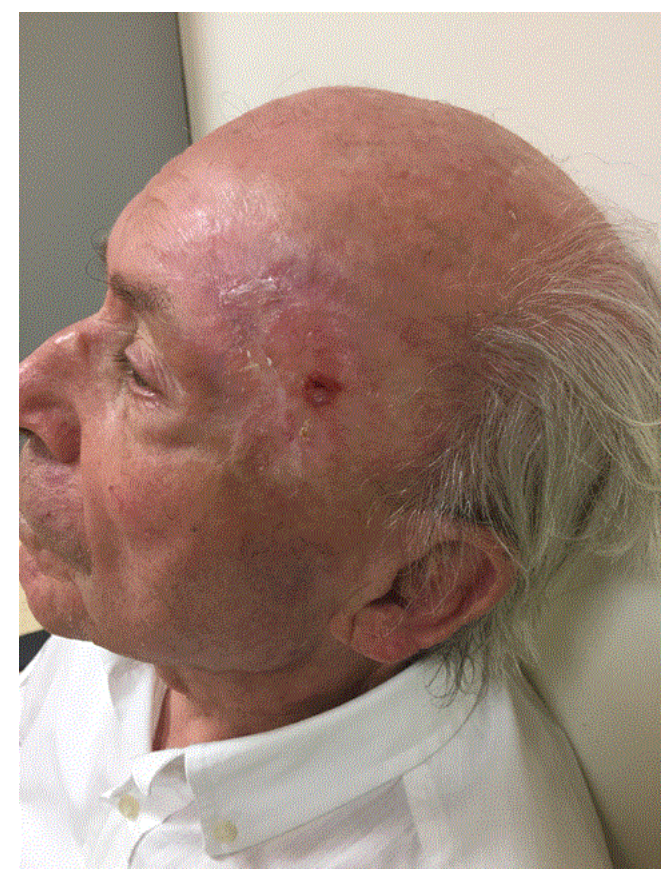

Figure 3: Toxicity and cosmetic result.

\section{Discussion}

The use of HDR BT in the treatment of skin cancer has been evaluated in several studies [8-10]. Compared to surgery and external beam RT, it is particularly suitable for elderly patients because of the shorter treatment, noninvasive nature and low toxicity [11].

The patients with BSC who are referred to RT often have unresectable tumors or comorbidities, the RT could be an effective therapeutic alternative for these patients with good rates of tumor control and a good cosmetic result [12].

Tumor local control with BT depends on a correct definition of margins and tumor volume. For the right definition of margins it is necessary to define the GTV and CTV. Most studies determine the GTV by delimitation of the clinically visible tumor and then adding 
$>1.5 \mathrm{~cm}$ margin to define the CTV for tumors larger than $2 \mathrm{~cm}$ [13-15].

In our work, based on the results of HDR-BT studies, margins of 2 $\mathrm{cm}$ for BSC would appear adequate.

Regarding the toxicity, some authors have showed that BT is associated with low toxicity and good cosmetic result $[12,16]$. In this case report, the patient tolerated the treatment well and he developed only skin acute toxicity grade 2 and late toxicity grade 1 with good cosmetic result.

The decision to prescribe 50 Gy in 20 fractions was based on the previous treatments, the patient's concern of significant side effects and the radical intent of treatment.

The hypofractionated regimen, also, allows an ambulatory treatment that reduces costs and treatment duration [13].

\section{Conclusion}

The scope of this case report is to discuss a procedure that unfortunately is utilized in a small amount of cases. However, it could be a valid alternative above all in selected elderly patients because it is a short treatment, with low toxicity, and lower costs. Furthermore, we shouldn't forget that BT doesn't utilize linear accelerators which can therefore be used in other patients.

HDR-BT could be an alternative option to external beam radiotherapy and surgery in selected frail patients with relapse of non melanoma cancer, because of the shorter treatment, noninvasive nature and low toxicity.

Randomized trials are needed to endorse these claims.

\section{References}

1. Aguilar Bernier M, Rivas Ruiz F (2012) Comparative epidemiological study of non-melanoma skin cancer between Spanish and north and central European residents on the Costa del Sol. J Eur Acad Dermatol Venerol 26: 41-47.

2. Wu TP, Stein JA (2013) Nonmelanoma skin cancer in young women. J Drugs Dermatol 12: 568-572.
3. http://www.nccn.org/professionals/physician_gls/f_guidelines.asp.

4. Bath-Hextall FJ, Perkins W, Bong J (2007) Interventions for basal cell carcinoma of the skin. Cochrane Database Syst Rev 24: CD003412.

5. Marín A, Vargas-Díez E, Cerezo L (2009) [Radiotherapy in dermatology]. Actas Dermosifiliogr 100: 166-181.

6. Dueck AC, Mendoza TR, Mitchell SA, Reeve BB, Castro KM, et al. (2015) Validity and Reliability of the US National Cancer Institute's PatientReported Outcomes Version of the Common Terminology Criteria for Adverse Events (PRO-CTCAE). JAMA Oncol .

7. Cox JD, Stetz J, Pajak TF (1995) Toxicity criteria of the Radiation Therapy Oncology Group (RTOG) and the European Organization for Research and Treatment of Cancer (EORTC). Int J Radiat Oncol Biol Phys 31: 1341-1346.

8. Liebmann A, Pohlmann S, Heinicke F, Hildebrandt G (2007) Helmet mold-based surface brachytherapy for homogeneous scalp treatment: a case report. Strahlenther Onkol 183: 211-214.

9. Rudoltz MS, Perkins RS, Luthmann RW, Fracke TD, Green TM, et al. (1998) High-dose-rate brachytherapy with a custom-surface mold to treat recurrent squamous cell carcinomas of the skin of the forearm. J Am Acad Dermatol 38: 1003-1005.

10. Cengiz M, Ozyar E, Ersu B, Akyol FH, Atahan IL (1999) High-dose-rate mold brachytherapy of early gingival carcinoma: a clinical report. J Prosthet Dent 82: 512-514.

11. Ballester-Sánchez R, Pons-Llanas O, Candela-Juan C, Celada-Alvarez FJ, de Unamuno-Bustos B, et al. (2015) Efficacy and safety of electronic brachytherapy for superficial and nodular basal cell carcinoma. J Contemp Brachytherapy 7: 231-238.

12. Nguyen NP, Ries T, Vock J, Vos P, Chi A, et al. (2015) Effectiveness of radiotherapy for elderly patients with non-melanoma skin cancer of the head. Geriatr Gerontol Int 15: 601-605.

13. Tormo A, Celada F, Rodriguez S, Botella R, Ballesta A, et al. (2014) Nonmelanoma skin cancer treated with HDR Valencia applicator: clinical outcomes. J Contemp Brachytherapy 6: 167-172.

14. Khan L, Choo R, Breen D, Assaad D, Fialkov J, et al. (2012) Recommendations for CTV margins in radiotherapy planning for non melanoma skin cancer. Radiother Oncol 104: 263-266.

15. Ballester Sánchez R, Pons Llanas O, Pérez Calatayud J, Botella Estrada R (2015) Dermoscopy margin delineation in radiotherapy planning for superficial or nodular basal cell carcinoma. Br J Dermatol 172: 1162-1163.

16. Bhatnagar A (2013) Nonmelanoma skin cancer treated with electronic brachytherapy: results at 1 year. Brachytherapy 12: 134-140. 